\title{
XX CONGRESSO BRASILEIRO DE ENGENHARIA QUÍMICA
}

\section{Título: Uso del gas pobre en motores de combustión interna para la generación de electricidad en Cuba.}

\author{
Autores: R. Lesme ${ }^{1}$, L. Garcia ${ }^{1}$, A. A. Recio ${ }^{1}$, L. O. Oliva ${ }^{1}$, J. A. Pajarín ${ }^{1}$, Yuniel Iglesias ${ }^{2}$. \\ ${ }^{1}$ Universidade de Oriente, Centro de Estudos de Eficiencia Energética, Cuba. \\ ${ }^{2}$ Empresa Forestal Integral Gran Piedra Baconao, Cuba. \\ Email: lesme1958@yahoo.es
}

\section{INTRODUCCIÓN.}

La biomasa ha sido presentada como una alternativa promisoria de energía, no sólo por su potencial mundial, sino porque es una fuente de $\mathrm{CO}_{2}$ neutral, su termo conversión genera bajas emisiones de $\mathrm{SO}_{2}$ y $\mathrm{NO}_{\mathrm{X}}$ y es la única fuente de energía renovable capaz de sustituir a los combustibles fósiles en todos sus mercados de energía.

Entre las fuentes de energía a partir de la biomasa los residuos de los aserraderos son particularmente atractivos económicamente, ellos están concentrados en un determinado depósito y sus costo de transportación es relativamente bajo. Se estima que durante el proceso de elaboración de la madera se obtienen $0.50 \mathrm{~m}^{3}$ de residuos $/ \mathrm{m}^{3}$ de madera en bolos (Lesme et al, 2009)

El gas pobre, resultado de la gasificación de biomasa con aire tiene un elevado contenido de sustancias volátiles, una alta estabilidad al encendido y puede ser utilizado en motores de combustión interna, el esquema tecnológico se presenta en la figura 1.

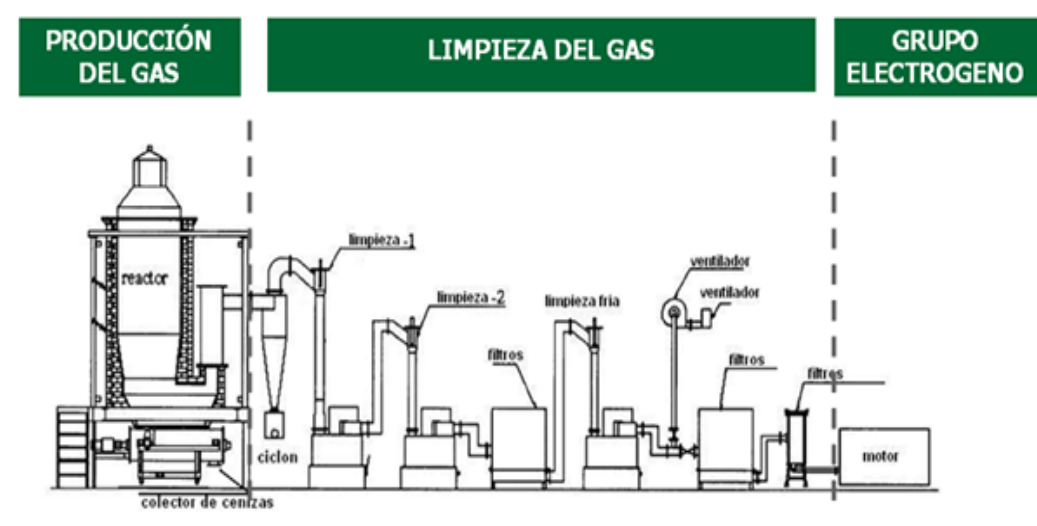

Figura 1. Esquema general de la tecnología.

El tipo de reactor depende fundamentalmente del rango de potencia eléctrica, de la cantidad y características de la biomasa y de la calidad del gas producto. Para la generación de electricidad a pequeña $(<1 \mathrm{MW})$ los más utilizados son los gasificadores downdraft, ellos son capaces de garantizar los requerimientos de calidad del gas a suministrar al motor. 


\section{CARACTERÍSTICAS DEL GAS. INFLUENCIA EN EL TRABAJO DEL MOTOR.}

Las características del gas que más influyen en el trabajo del motor son: Composición, densidad energética de la mezcla aire-gas pobre, número de metano y velocidad de la llama.

\subsection{Composición del gas pobre.}

En un gasificador, la biomasa puede ser convertida en un gas compuesto por $\mathrm{H}_{2}, \mathrm{CO}$, $\mathrm{CO}_{2}, \mathrm{H}_{2} \mathrm{O}, \mathrm{CH}_{4}$, alquitrán y particulados. La composición exacta depende de las características de la biomasa, de las condiciones de operación y el tipo de reactor. (Martinez et al, 2011)

\subsection{Densidad energética}

Tomando en cuenta que los motores a gas trabajan aproximadamente en condiciones estequiométricas de combustión, la densidad energética de la mezcla puede ser determinada por la siguiente expresión: (Sridhar et al 2001).

$$
\mathrm{HV}_{\mathrm{m}}=\frac{10768\left(\mathrm{Y}_{\mathrm{H}_{2}}\right)+12696\left(\mathrm{Y}_{\mathrm{CO}}\right)+35866\left(\mathrm{Y}_{\mathrm{CH}_{4}}\right)}{1+2.38\left(\mathrm{Y}_{\mathrm{H}_{2}}\right)+2.38\left(\mathrm{Y}_{\mathrm{CO}}\right)+9.53\left(\mathrm{Y}_{\mathrm{CH}_{4}}\right)}
$$

Donde:

$\mathrm{HV}_{\mathrm{m}}$. Densidad energética de la mezcla aire-gas pobre, $\mathrm{kJ} / \mathrm{Nm}^{3}$

$\mathrm{Y}_{\mathrm{H} 2}, \mathrm{Y}_{\mathrm{CO}}, \mathrm{Y}_{\mathrm{CH} 4}$. Concentraciones de hidrógeno, monóxido de carbono y metano en el gas.

\subsection{Número de metano.}

El número de metano es un indicador de los combustibles gaseosos que mide su tendencia a la detonación, mientras mayor sea su valor, menor la tendencia. Existe una escala denominada número de metano $(\mathrm{NM})$ que utiliza como referencias el metano puro $(\mathrm{NM}=$ 100) y el hidrógeno $(\mathrm{NM}=0)$ que valora la aplicabilidad del gas en los motores, considerándose un valor entre 75-80 como satisfactorio (Przybyla G et al, 2010)

\subsection{Velocidad de la llama y ángulo de avance.}

La velocidad de la llama es un parámetro que define las características de oxidación de un combustible, su valor dependen de su composición química, de la cantidad de aire empleada para su combustión, así como, las condiciones de presión y temperatura de la mezcla. Es posible el uso de un combustible en motores de combustión interna cuando la velocidad de la llama está por encima de $8 \mathrm{~cm} / \mathrm{s}$ en condiciones estequiométricas de combustión.

El ángulo de avance de la ignición está íntimamente ligado a la velocidad de la llama, el mismo deberá ser atrasado cuando la mezcla presente una elevada velocidad, con el fin de alcanzar mayores potencias y eficiencias. ( $\mathrm{J}$ et al, 2006)

En la tabla 1 se presentan la densidad energética, el número de metano y velocidad de la llama del gas pobre, como puede observarse de acuerdo a los valores del número de metano y velocidad de la llama su empleo en motores resulta satisfactorio. 
Tabla 1. Densidad energética, número de metano y velocidad de la llama del gas natural y el gas pobre. (Muñoz, 2000)

\begin{tabular}{|c|c|c|}
\hline Indicadores & Gas Natural & Gas Pobre \\
\hline Densidad Energética de la mezcla, $\left(\mathrm{MJ} / \mathrm{Nm}^{3}\right)$ & 3.32 & 2.20 \\
\hline Número de metano & 105 & 80 \\
\hline Velocidad de la llama, cm/s & 35 & 50 \\
\hline
\end{tabular}

\section{ESTADO DEL ARTE DE LOS MOTORES DE COMBUSTIÓN INTERNA ALIMENTADOS CON GAS POBRE.}

Las aplicaciones comerciales de la tecnología se localizan en un rango de potencia menor de $1 \mathrm{MW}$. Los motores utilizados pueden ser de ignición por chispa y Diesel. En los motores de ignición por chispa el gas pobre puede sustituir el 100\% del combustible. La principal modificación a realizar consiste en el atraso del tiempo de ignición a fin de lograr que la presión máxima de combustión se alcance entre 10 y 12 grados DPMS y con ello mejores desempeños del motor (Sridhar et al, 2001)

Los motores Diesel operan a mayores relaciones de compresión, su operación con gas pobre debe ser dual (sustitución de hasta el 85\% del Diesel). La ventaja radica en su flexibilidad, en caso de un mal funcionamiento del gasificador es posible un cambio inmediato de combustible, operando el motor totalmente con Diesel. Cuando se quiere utilizar un motor Diesel con gas pobre (100\%) hay que implementar un mezclador y un sistema de ignición por chispa y modificarse el ángulo de avance de la ignición (Ramadhas et al 2008)

En los últimos años varios trabajos se han reportado sobre la evaluación del desempeño de los motores accionados con gas pobre, en la tabla 2 se ofrecen algunos resultados.

Tabla 2- Motores de combustión interna trabajando con gas pobre.

\begin{tabular}{|c|c|c|c|c|c|c|c|c|c|c|}
\hline Motor & rpm & RC & $\begin{array}{l}\text { Gas. } \\
(\%)\end{array}$ & Mod. & $\begin{array}{l}\text { Pot } \\
(\mathrm{kW})\end{array}$ & $\begin{array}{c}\text { PI } \\
\text { (PMS) }\end{array}$ & $\begin{array}{l}\text { Pp } \\
(\%)\end{array}$ & $\begin{array}{l}\eta_{\mathrm{T}} \\
(\%)\end{array}$ & $\begin{array}{l}\eta_{G} \\
(\%)\end{array}$ & Ref. \\
\hline Diesel & 1500 & 11.5:1 & 100 & SIG & $12-16^{(1)}$ & 35 & nd & $28-32$ & $21-24^{(2)}$ & $\begin{array}{l}\text { Shashhika } \\
\text { ntha,et } \\
\text { al,1993 }\end{array}$ \\
\hline Diesel & 1500 & $17: 1$ & 100 & SIG & $2.3^{(3)}$ & 10 & 20 & $19.05^{(4)}$ & nd & $\begin{array}{l}\text { Ramachan } \\
\text { dra, } 1993\end{array}$ \\
\hline Diesel & nd & nd & 100 & SIG & $15-20^{(1)}$ & $\mathrm{nd}$ & 20 & $28^{(4)}$ & $25^{(2)}$ & $\begin{array}{l}\text { Henriskse } \\
\mathrm{n} \quad \text { et al, } \\
2006\end{array}$ \\
\hline Otto & 1800 & 12:1 & 100 & MGN & $40.7^{(4)}$ & 30 & 37.38 & nd & nd & $\begin{array}{l}\text { Muraro, } \\
2006\end{array}$ \\
\hline Diesel & 1500 & 18.5:1 & 81 & No & $11.44^{(3)}$ & nd & 21 & $14.7^{(5)}$ & $11.69^{(2)}$ & $\begin{array}{l}\text { Bhattachat } \\
\text { ya et al, } \\
2001\end{array}$ \\
\hline Diesel & 1500 & $17: 1$ & 100 & SIG & $17.5^{(1)}$ & 6 & $16.7^{(6)}$ & $\mathrm{nd}$ & $16.6^{(7)}$ & Shridha \\
\hline
\end{tabular}




\begin{tabular}{|c|c|c|c|c|c|c|c|c|c|c|}
\hline & & & & & & & & & & Gr, 2001 \\
\hline \multirow[t]{2}{*}{ Diesel } & \multirow[t]{2}{*}{1500} & \multirow[t]{2}{*}{$17.5: 1$} & 65 & No & \multirow[t]{2}{*}{$4^{(3)}$} & \multirow[t]{2}{*}{27} & \multirow[t]{2}{*}{20} & 27 & nd & \multirow{2}{*}{$\begin{array}{l}\text { Ahrefeldt } \\
\text { et al, } 2000\end{array}$} \\
\hline & & & 60 & SIG & & & & 24 & nd & \\
\hline Diesel & 1800 & $12.5: 1$ & 100 & SIG & $5.3^{(1)}$ & 15 & 47 & nd & nd & $\begin{array}{l}\text { Viera } \mathrm{R} \text { et } \\
\text { al, } 2011\end{array}$ \\
\hline Gás & 1500 & $12: 1$ & 100 & No & 75 & 28 & 14 & 20.7 & - & $\begin{array}{c}\text { Raman P } \\
\text { et al, } \\
2013\end{array}$ \\
\hline
\end{tabular}

(1) Potencia eléctrica, nd. No declarado, (2) Biomasa-electricidad, (3) Potencia al freno, (4) En el eje, (5) Motor-generador, (6) Potencia mecánica, (7) Biomasa-salida en el eje, RC. Relación de Compresión, Mod. Modificaciones en el motor, SIG. Sistema de ignición, PI. Punto de ignición, APMS. Antes del punto muerto superior, $\eta_{\mathrm{T}}$. Eficiencia Térmica, $\eta_{\mathrm{G}}$. Eficiencia Global.

\section{EVALUACIÓN DE UN MOTOR ASHOK LEYLAND TRABAJANDO CON GAS POBRE. MATERIALES Y MÉTODOS.}

\subsection{Equipamiento.}

El gas es producido en un reactor downdraft (Figura 2), modelo COMBO-80, (Owner's Manual, 2009)

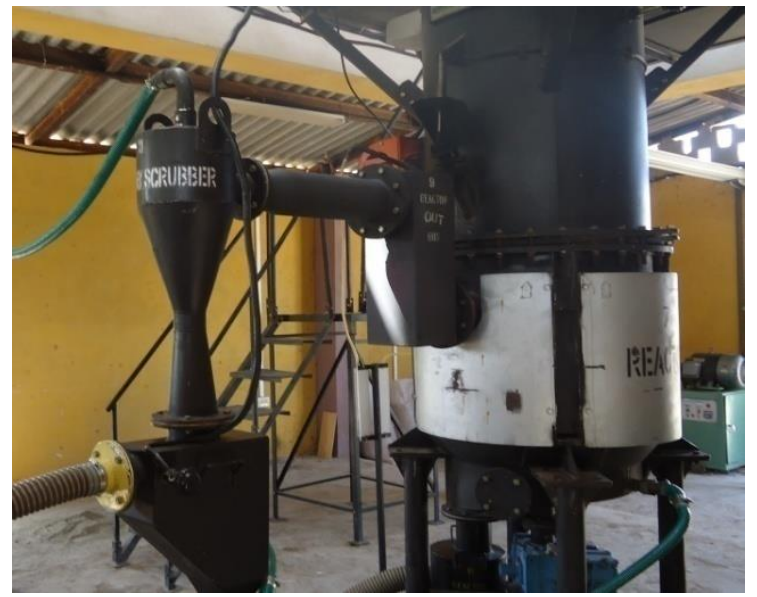

Figura 2- Gasificador downdraft

El gas se somete a un proceso de limpieza en un sistema compuesto por un filtro grueso, un filtro fino activo y otro pasivo, y un filtro de seguridad (Figura 3.) 


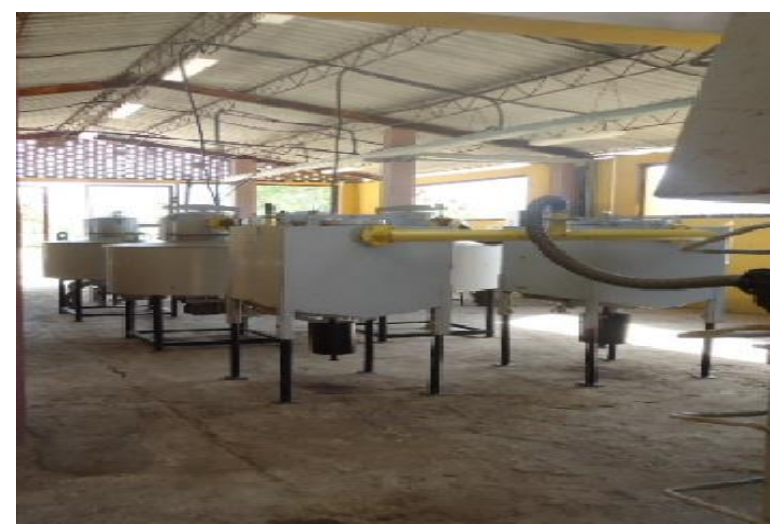

Figura 3- Sistema de limpieza seca.

La unidad generadora está formada por un motor y un generador eléctrico. El generador, directamente acoplado al motor, es trifásico, gira a $1800 \mathrm{rpm}$ de $220 \mathrm{~V}$ y $60 \mathrm{~Hz}$. (Figura 4)

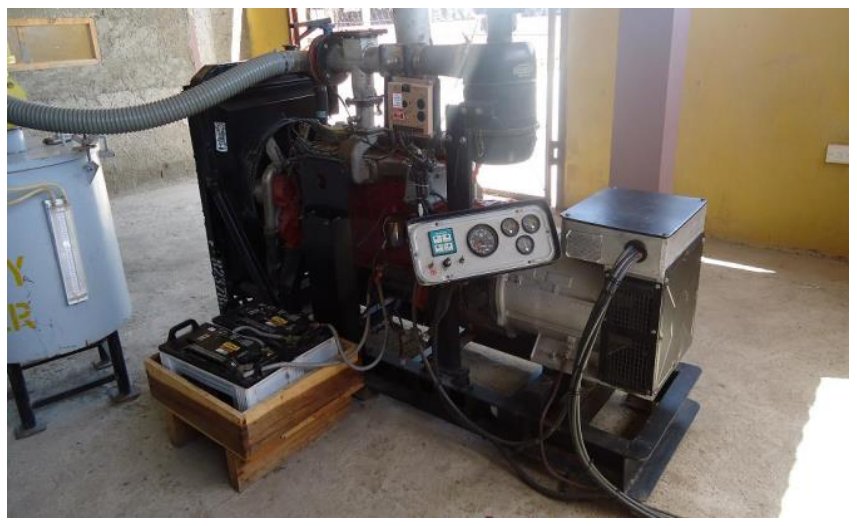

Figura 4. Unidad generadora de electricidad.

El motor Diesel modelo Leyland fue modificado para trabajar con gas pobre mediante la implementación de un mezclador tipo Venturi y un sistema de encendido por chispa eléctrica, sus características técnicas aparecen en la "Tabla 3" (Leyland P76, 1973)

Tabla 3- Características técnicas del motor.

\begin{tabular}{|c|c|}
\hline Modelo & Leyland \\
\hline Revoluciones & 1800 \\
\hline No. de Cilindros & 6 \\
\hline Cilindrada & $2623 \mathrm{~cm}^{3}$ \\
\hline Diámetro del pistón & $76,2 \mathrm{~mm}$ \\
\hline Carrera & $95.7 \mathrm{~mm}$ \\
\hline Potencia Nominal & $90.2 \mathrm{~kW}($ Diesel) \\
\hline Relación de compresión & $9: 1$ \\
\hline
\end{tabular}




\subsection{Técnica Experimental.}

El motor se evaluó para tres condiciones de trabajo, en cada caso se midió la carga eléctrica y el flujo de gas suministrado al motor. La carga eléctrica a través de un Watímetro situado en el panel de control y el flujo de gases con un tubo Venturi ( $\left.\mathbf{M}_{\text {gases }}\right)$ situado a la entrada del motor.

La composición del gas y el consumo de biomasa del gasificador $\left(\mathrm{M}_{\text {bio }}\right)$ se obtuvieron mediante un modelo de equilibrio termodinámico no estequiométrico del gasificador. Los resultados de las pruebas y el modelo se recogen en la "Tabla 4" ( Trabajo de Grado, 2013)

Tabla 4. Mediciones experimentales y resultados del modelo termoquímico.

\begin{tabular}{|c|c|c|c|c|c|c|c|}
\hline No & \multicolumn{7}{|c|}{ Potencia Eléctrica $\left(P_{\text {ele }}\right)=5 \mathrm{~kW}$} \\
\hline 1 & \multicolumn{5}{|c|}{ Composición del gas, (\%v) } & \multirow{2}{*}{$\begin{array}{l}M_{\text {gases }} \\
\text { (kg/h) }\end{array}$} & \multirow{2}{*}{$\begin{array}{c}M_{\text {bio }} \\
(\mathrm{kg} / \mathrm{h})\end{array}$} \\
\hline 2 & $\mathrm{CO}$ & $\mathrm{CO}_{2}$ & $\mathrm{CH}_{4}$ & $\mathrm{~N}_{2}$ & $\mathrm{H}_{2}$ & & \\
\hline 3 & 21 & 13 & 3 & 46 & 16 & 56.32 & 29.48 \\
\hline No & \multicolumn{7}{|c|}{ Potencia Eléctrica $\left(P_{\text {ele }}\right)=20 \mathrm{~kW}$} \\
\hline 1 & \multicolumn{5}{|c|}{ Composición del gas, (\%v) } & $\mathbf{M}_{\text {gases }}$ & $\mathbf{M}_{\text {bio }}$ \\
\hline 2 & $\mathrm{CO}$ & $\mathrm{CO}_{2}$ & $\mathrm{CH}_{4}$ & $\mathrm{~N}_{2}$ & $\overline{\mathrm{H}_{2}}$ & $(\mathbf{k g} / \mathbf{h})$ & $(\mathrm{kg} / \mathrm{h})$ \\
\hline 3 & 20 & 12 & 2 & 46 & 18 & 7.7 .33 & 30.28 \\
\hline No & \multicolumn{7}{|c|}{ Potencia Eléctrica $\left(P_{\text {ele }}\right)=40 \mathrm{~kW}$} \\
\hline 1 & \multicolumn{5}{|c|}{ Composición del gas, (\% v) } & $\mathbf{M}_{\text {gases }}$ & $\mathbf{M}_{\text {bio }}$ \\
\hline 2 & $\mathrm{CO}$ & $\mathrm{CO}_{2}$ & $\mathrm{CH}_{4}$ & $\mathrm{~N}_{2}$ & $\mathrm{H}_{2}$ & $(\mathbf{k g} / \mathbf{h})$ & $(\mathrm{kg} / \mathrm{h})$ \\
\hline 3 & 19 & 10 & 2 & 50 & 18 & 155.03 & 60.00 \\
\hline
\end{tabular}

\section{RESULTADOS.}

\subsection{Eficiencia térmica del motor.}

Evalúa el proceso de transformación de energía de la mezcla combustible en electricidad.

$$
\eta_{\mathrm{T}}=\frac{\text { Potencia Eléctrica }}{\mathrm{HV}_{\mathrm{M}} \mathrm{xM}}
$$

Donde:

$\mathrm{M}_{\text {mezcla. }}$. Flujo volumétrico de la mezcla, $\mathrm{Nm}^{3} / \mathrm{s}$

El flujo volumétrico de la mezcla se determinó por la expresión (3) (Trabajo de Grado, 2013)

$$
\mathrm{M}_{\text {mezcla }}=\mathrm{M}_{\text {gases }}+\mathrm{M}_{\text {aire }}
$$

La masa de aire $\left(\mathrm{M}_{\text {aire }}\right)$ se determinó través de la expresión general de la combustión del gas pobre, teniendo en cuenta su composición y los resultados de balance molares de cada uno de los elementos que lo conforman. (Trabajo de grado, 2013) 


\subsection{Eficiencia global de la instalación.}

Evalúa el proceso de transformación de la energía de la biomasa en electricidad (Ec. 4)

$$
\eta_{\mathrm{G}}=\frac{\text { Potencia Eléctrica }}{\mathrm{M}_{\mathrm{bio}} \mathrm{XPCI}_{\mathrm{bio}}}
$$

Donde

$\mathrm{PCI}_{\mathrm{bio}}$. Poder calórico bajo de la biomasa, $\mathrm{kJ} / \mathrm{kg}$

Los resultados de los cálculos se reportan en la "Tabla 5"

Tabla 5. Eficiencia del motor y la instalación.

\begin{tabular}{|c|c|c|c|c|}
\hline $\begin{array}{c}\mathrm{P}_{\text {ele }} \\
(\mathrm{kW})\end{array}$ & $\begin{array}{c}\mathrm{HV}_{\mathrm{m}} \\
\left(\mathrm{MJ}^{3} \mathrm{Nm}^{3}\right)\end{array}$ & $\begin{array}{c}\mathrm{M}_{\text {mezcla }} \\
\left(\mathrm{Nm}^{3} / \mathrm{s}\right)\end{array}$ & $\begin{array}{c}\eta_{\mathrm{T}} \\
(\%)\end{array}$ & $\begin{array}{c}\eta_{\mathrm{G}} \\
(\%)\end{array}$ \\
\hline 5 & 2.05 & 0.024 & 10.2 & 3.6 \\
\hline 20 & 2.45 & 0.033 & 24.0 & 14.0 \\
\hline 40 & 2.40 & 0.065 & 25.7 & 14.2 \\
\hline
\end{tabular}

Los resultados muestran una eficiencia térmica del motor entre 10,2 y 25,7\% manteniéndose de forma estable en un amplio rango de carga $(20-40 \mathrm{~kW})$

\section{CONCLUSIONES}

1. El gas pobre presenta propiedades combustibles inferiores a la del gas natural (densidad energética y número de metano), no obstante puede ser utilizado en motores de combustión interna de forma satisfactoria, esperándose una reducción de la potencia del motor con respecto a los combustibles tradicionales proporcional a la disminución de su densidad energética. En el caso del motor Leyland P76, la reducción fue de $42 \%$ con respecto al Diesel.

2. La mayor parte de los motores utilizados para trabajar con gas pobre son Diesel a los cuales se le implementa un mezclador, un sistema de ignición por chispa y se modifica el ángulo de avance.

3. La evaluación del desempeño de los motores de combustión interna accionados con gas pobre para la generación de electricidad a pequeña escala, muestran que ellos trabajan con una eficiencia térmica en un rango de 20-32\% y una eficiencia global entre $12-25 \%$. En el caso del motor Leyland P76 que trabaja con gas pobre producto de la gasificación de residuos de la industria forestal posee una eficiencia térmica de $25.7 \%$ y una eficiencia global de $14.2 \%$.

\section{BIBLIOGRAFÍA.}

1. Ahrenfeldt, J., Henriksen, U., Schramm, J. Experimental on Wood Gas Engines. The Technical University of Denmark. Department of Energy Engineering, 40 p. Año 2000 
2. Bhattachatya, S. C., Hla, S. S., Pham, H. L. "A Study on a Multi- Stage Hybrid Gasifier-Engine System", Biomass and Bioenergy, vol. 21, pp. 445-460. Año 2001

3. Henriksen, U., Ahrenfeldt, J., Jensen, T, Gobel, B, Bentzen, J, Hindsgaul, C, Sorensen, L.H. "The Design, Construction and Operation of a 75 kW Two-Stage Gasifier", Energy, vol. 31, pp. 1542-1553. Año 2006

4. Lesme Jaén R, Oliva Ruiz L. Potencial energético de los residuos de la industria de la madera en el aserrío "El Brujo" de la Empresa Gran Piedra Baconao. The 8th latinamerican congress on electricity generation and transmission - CLAGTEE 2009

5. Leyland P76, 1973 especificaciones técnicas 257884

6. Martínez D. J, Viera Andrade R, Silva Lora E, Lesme Jaén R. Experimental study on biomass gasification in a double air stage downdraft reactor. Revista: Biomass and Bioenergy XXX. Pag.1-17. Año 2011.

7. Muñoz M., Moreno, F., Morea-Roy, J., Ruiz J., Arauzo, J. "Low Heating Value Gas on Spark Ignition Engines", Biomass and Bioenergy, vol. 18, pp. 431-439. Año 2000.

8. Muraro, W. Avaliação do Funcionamento de Motor ICE com Gás de Baixo Poder Calorífico Proveniente da Gaseificação de Casca de Arroz, Dissertação de Mestrado, Universidade Estadual de Campinas, UNICAMP, 86p. Año 2006

9. Owner's Manual. Ankur Scientific Energy Technologies Pvt. Ltd. ISO 9001 / ISO 14001 / BS OHSAS 18001 Certified Company. Año 2009.

10. Ramadhas A, Jayaraj, Muraleedharan C. Dual fuel mode operation in diesel engines using renewable fuels: Rubber seed oil and coir-pith producer gas. Renewable Energy No. 33 PP. 2077-2083. Año 2008.

11. Ramachandra, A. "Performance Studies on a Wood Gas Run IC Engine", Recent Advances in Biomass Gasification and Combustion. Proceedings of Fourth National Meet on Biomass Gasification and Combustion, pp. 213-218. Año 1993

12. Raman P, N.K. Ram N, Gupta R. A dual fired downdraft gasifier system to produce cleaner gas for power generation: Design, development and performance analysis. Energy 54 (2013) 302-314.

13. Shashikantha, B, Khairnar P, Kamat, Parikh P. Development and Performance Analysis of a $15 \mathrm{kWe}$ Producer Gas Operated SI Engine. Recent Advances in Biomass Gasification and Combustion. Proceedings of Fourth National Meet on Biomass Gasification and Combustion,. pp. 219-231. Año 1993

14. Sridhar G, Paul J, Mukunda H. Biomass derived producer gas as a reciprocating engine fuel—an experimental analysis. Biomass and Bioenergy No. 21 Año (2001) PP. 61-72.

15. Trabajo de Grado. Evaluación teórico experimental de un gasificador downdraft modelo COMBO-80. Centro de Estudios de Eficiencia Energética. Universidad de Oriente. Santiago de Cuba. Año 2013.

16. Trabajo de Grado. Evaluación Termodinámica del Motor Ashok Leyland LTD alimentado con gas pobre. Centro de Estudios de Eficiencia energética. Universidad de Oriente. Santiago de Cuba. Año 2013.

17. Vieira R, Silva E, Lesme R. Análise da operação de um gaseificador co-corrente de duplo estágio acoplado a um motor de combustão interna de ignição por centelha. Congreso Iberoamericano de Ingeniería Mecánica. CIBIM 2011. Porto Potugal. Año 2011 